\title{
Plate wastage and nutritional intake in inpatients with renal disease: a comparison of bulk trolley and kitchen plated food services
}

\author{
D. Cheeseman ${ }^{1}$, T. P. Mitchell ${ }^{2}$, Y. M. Jeanes ${ }^{1}$, V. Heard ${ }^{3}$ and K. Michie ${ }^{3}$ \\ ${ }^{1}$ School of Human \& Life Sciences, Roehampton University, London SW15 4JD, UK, ${ }^{2}$ Department of Health and Human \\ Sciences, London Metropolitan University 166-220 Holloway Road, London N7 8DB, UK and ${ }^{3}$ Department of Nutrition \\ and Dietetics, Guy's and St Thomas' NHS Foundation Trust, Great Maze Pond, London SE1 9RT, UK
}

Patients with renal disease are often malnourished $(30-76 \%)$ and poor dietary intake is frequently reported in this patient group ${ }^{(1)}$. The method of food delivery to inpatients has been shown to influence food intake ${ }^{(2)}$, thus having the potential to impact on malnutrition. The aim of the present study was to determine whether a bulk trolley food delivery service resulted in improved nutritional intake and reduced plate wastage when compared with a kitchen plated service.

The study took place on two renal wards in a London teaching hospital over two 1-week periods (November 2006 and June 2007); the menu options offered were the same on each week and midday and evening meals were included. Food returned to the kitchen was weighed for each service.

The meals served to the patients were of a similar size, independent of the food delivery service. However, significantly more food was consumed with the bulk trolley compared with the plated service. Patients consumed significantly more energy with the bulk service. The range of food intake for both services was large, from $100 \%$ eaten to none eaten.

\begin{tabular}{|c|c|c|c|c|c|c|c|c|c|c|}
\hline & \multicolumn{2}{|c|}{ Food served wt (g) } & \multicolumn{2}{|c|}{ Food wastage (\%) } & \multicolumn{2}{|c|}{ Food eaten wt $(\mathrm{g})$} & \multicolumn{2}{|c|}{ Energy intake (kJ) } & \multicolumn{2}{|c|}{ Protein intake $(\mathrm{g})$} \\
\hline & Mean & SD & Mean & SD & Mean & SD & Mean & SD & Mean & SD \\
\hline Plated service ( 65 meals) & 531 & 203 & 45 & 24 & 288 & 158 & 1331 & 628 & 17 & 12 \\
\hline Bulk service ( 85 meals) & 515 & 159 & 29 & 26 & 362 & 172 & 1657 & 787 & 20 & 14 \\
\hline Significance* & \multicolumn{2}{|c|}{ NS } & \multicolumn{2}{|c|}{$P<0.001$} & \multicolumn{2}{|c|}{$P=0.007$} & \multicolumn{2}{|c|}{$P=0.005$} & \multicolumn{2}{|c|}{ NS } \\
\hline
\end{tabular}

*Determined using an independent $t$ test.

Protein intakes were similar for both services. Significantly less carbohydrate and fat were consumed with the plated service compared with the bulk service (carbohydrate 36 (SD 23) v. 47 (SD 24) g, $P=0.01$; fat 13 (SD 10) v. 16 (SD 10) g, $P=0.05$ ). From a catering perspective, although the amount of food wasted was less with the bulk service, it remained above the recommendations of $10 \%{ }^{(3)}$. The present study is limited by inherent differences at the time of data collection including the time of year and different inpatients.

The findings in the current study are similar to previous findings ${ }^{(2)}$. It is important to audit the change in food delivery service to inpatients in order to determine whether improvements have occurred. The bulk service resulted in increased energy intake and reduced plate wastage compared with plated service in inpatients with renal disease. Further research is required to determine whether such changes in food service delivery result in changes in clinical outcome.

1. Akner G \& Cederholm T (2001) Am J Clin Nutr 74, 6-24.

2. Marson H, McErlain L \& Ainsworth P (2003) Br Food J 105, 791-799.

3. Department of Health (1996) Hospital Catering Delivering a Quality Service. London: NHS Executive. 